\title{
Laboratory Test Character Result in Standard Unit
}

National Cancer Institute

\section{Source}

National Cancer Institute. Laboratory Test Character Result in Standard Unit. NCI

Thesaurus. Code C83319.

The standard character or string for representation and reporting of laboratory test data. 